\title{
Incarceration of the Ulnar Nerve after Dislocated Elbow Fracture: A Case Report and Review of the Literature
}

\author{
Mohamed Rida El Galiou , Houass EY, Boufettal M, Bassir RA, Kharmaz M, Lamrani MO, Berrada MS
}

Department of Orthopedics and Traumatology, University Hospital Center of Rabat, Faculty of Medicine and Pharmacy of Rabat, University Mohammed V Souissi, Rabat, 10000, Morocco

DOI: $10.36347 /$ sjams.2020.v08i11.033

| Received: 09.11.2020 | Accepted: 21.11.2020 | Published: 23.11.2020

*Corresponding author: Mohamed Rida El Galiou

Abstract

Case Report

Incarceration of the ulnar nerve after elbow dislocation is a rare complication. The authors report a case of ulnar nerve incarceration over a dislocated elbow fracture. This is a 17-year-old patient attending the emergency room for a fractured dislocated elbow without vascular nerve damage. Faced with the incoercibility of the dislocation, a bloody reduction was decided. The exploration objectified the incarceration of the ulnar nerve. After extrication, the epitrochlea was fixed with two pins and the anterior transposition of the ulnar nerve.

Keywords: Elbow dislocation, ulnar nerve, Incarceration.

Copyright $($ C 2020 The Author(s): This is an open-access article distributed under the terms of the Creative Commons Attribution 4.0 International License (CC BY-NC 4.0) which permits unrestricted use, distribution, and reproduction in any medium for non-commercial use provided the original author and source are credited.

\section{INTRODUCTION}

Incarceration of the ulnar nerve after elbow dislocation is a rare complication. We report a case of ulnar nerve incarceration in a postero-external dislocation of the elbow.

\section{Clinical Observation}

This is a 17-year-old, right-handed, victim of closed trauma to the left elbow. At the physical examination we noted: a swollen elbow, deformed with loss of anatomical landmarks, painful on mobilization and palpation leading to total functional impotence, an absence of sensory and motor deficit of the upper limb concerned and the distal pulses were well perceived. The standard X-ray showed a postero-external dislocation of the elbow associated with a fracture of the epitrochlea (fig. 1). Reduction under anesthesia was attempted without results and without post-reduction ulnar palsy. Faced with the incoercibility of the dislocation and the risk of lesions that these manipulations could induce, closed reduction is abandoned and emergency intervention is decided. Internally, facing the medial epicondyle, exploration demonstrated the incarceration of the ulnar nerve (fig. 2 ), the epitrochlear muscles and the epitrochlea. After careful extrication of the muscle group returning the epitrochlea, facilitated by the hypervalgus maneuver, the epitrochlea was fixed by two pins and the anterior transposition of the ulnar nerve. Radiographic control was satisfactory, showing perfect reduction (fig. 3). The elbow is immobilized in a brachioantébrachio-palmar splint at $90^{\circ}$ elbow flexion and pronation for four weeks. The evolution was marked by the stability of the elbow, consolidation of the fracture and the absence of nervous deficit.

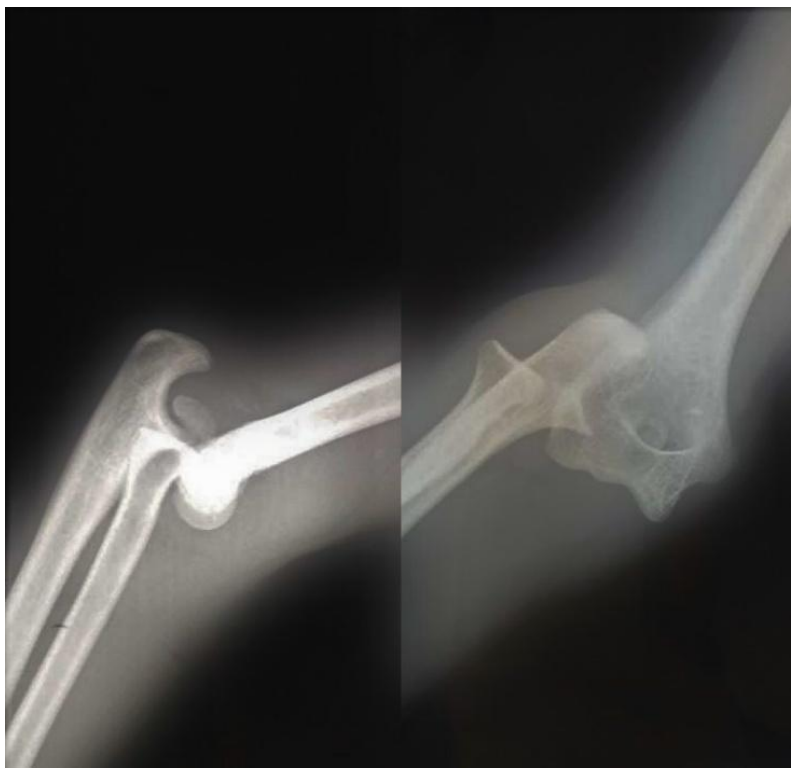

Fig-1: X-ray of the elbow, face and profile showing a postero-external dislocation of the elbow associated with a fracture of the epitrochlea 


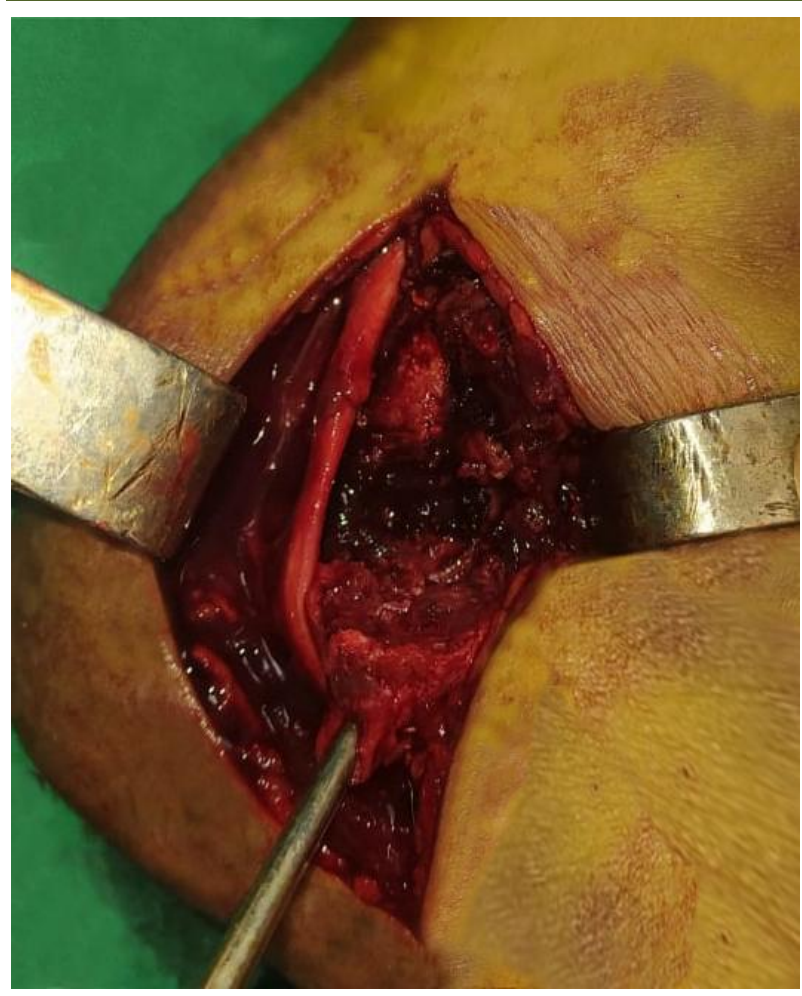

Fig-2: Intraoperative view showing the incarceration of the ulnar nerve

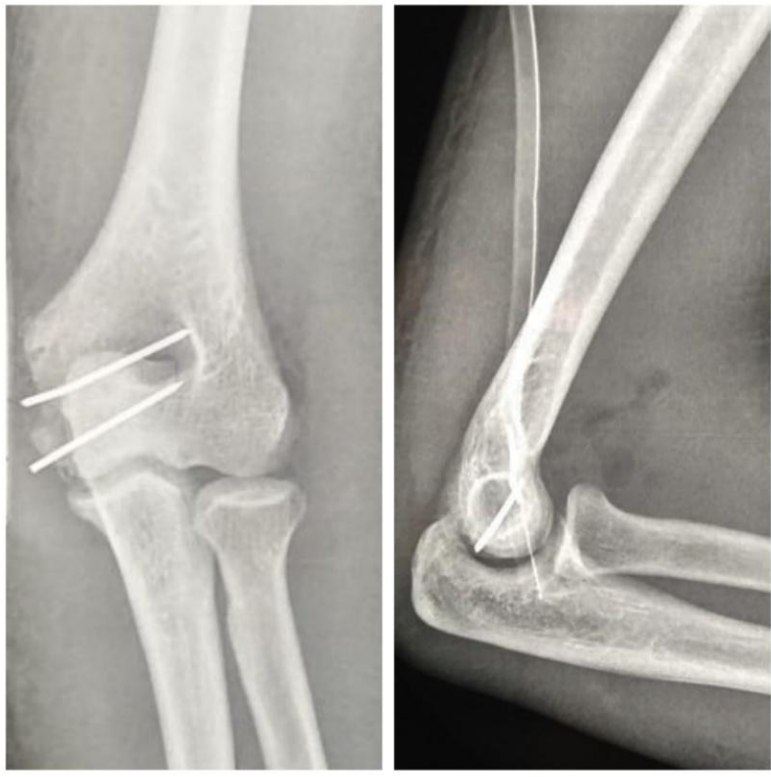

Fig-3: Control x-ray of the elbow, face and profile, showing reduction in dislocation and pinning of the epitrochlear

\section{DiscUSSION}

Elbow dislocation is the primary dislocation of a major joint in children, and the second in adults, frequently occurring in sports accidents [1]. Medial epicondyle fracture is the most common complication of elbow dislocation, but unfortunately often goes unnoticed [1]. It is found mainly in adolescents where it takes the form of an apophyseal tear linked to the area of weakness represented by the growth cartilage not yet fused at this level. It is not uncommon for this avulsed epicondylar fragment to end up incarcerated in the humero-ulnar space. It is therefore important to differentiate it from a humeral epiphyseal nucleus because this intra-articular displacement requires surgical reduction of the dislocation by arthrotomy [2]. Surgical fixation of the epicondyle is indeed essential because it is the area of insertion of the ulnar collateral ligament, whose role in the stability of the elbow joint is essential [2].

Nerve complications involve the ulnar nerve $[3,4]$ and the median nerve $[5,6]$. These two trunks can be injured during the trauma by stretching or after reduction of the dislocation by incarceration. Forced reduction movements should be avoided in order to avoid the incarceration of the nerve and the persistence of a sensory and / or motor deficit in the territory of the ulnar nerve.

Tropet et al. [7] summarizes the mechanism of nervous incarceration during dislocations of the elbow, specifying the two essential elements that occur in the pathogenesis of incarceration:

*On the one hand, a musculo-ligamentous disinsertion or an epitrochlear fracture, allowing the engagement of the nerve in the joint;

* On the other hand, incarceration occurs only during reduction, justified by the absence of neurological disorders before reduction.

The observation of nerve damage requires emergency orthopedic reduction when the paralysis is contemporaneous with the trauma and surgical exploration if it occurs during the reduction [8]. For our patient we opted for an anterior transposition of the ulnar nerve. The anterior transposition of the ulnar nerve can be subcutaneous, intramuscular or submuscular $[9,10]$.

\section{CONCLUSION}

Incarceration of the ulnar nerve after elbow dislocation is a rare complication. Before any incoercible dislocation of the elbow, possible incarceration of the fragments and / or soft parts should be sought. Blood reduction is necessary for the release and transposition of the nerve.

\section{REFERENCE}

1. Parsons BO, Ramsey ML. Acute elbow dislocations in athletes. Clinics in sports medicine. 2010 Oct;29(4):599-609.

2. Tourdias D. Luxation du coude compliquée d'une fracture-avulsion de l'épicondyle médial chez un enfant. Annales françaises de médecine d'urgence. 2015 Jan 1;5(1):50- 
3. Grobler GP. Unusual cause of ulnar nerve Palsy. ClinOrthop. 1996; 323: 192-193

4. Linscheid RL, O'Driscoll SW. Elbow dislocations. In: Morrey BF ed. The elbow and its disorders. Philadelphia: WB Saunders. 1993 : 441-452

5. Limb D, Hodkinson SL, Brown RF. Median nerve palsy after posterolateral elbow dislocation. J Bone Joint Surg. 1994; 76B : 987-988

6. Rao SB, Crawford AH. Median nerve entrapment after dislocation of the elbow in children. Clin Orthop. 1995; 312 : 232-237
7. Tropet Y, Menez D, Breintini JM, Vichard Ph. Incarcération du nerf médian après une luxation du coude. Acta OrthopBelg. 1989;55:217-21

8. Bégué T. Luxations du coude. Encyclopédie médico-chirurgicale.14-042-A-10

9. Childress HM. Recurrent ulnar-nerve dislocation at the elbow. ClinOrthop. 1975; 108: 169-173.

10. Rayan GM. Recurrent anterior dislocation of the ulnar nerve at the cubital tunnel. Plast ReconstrSurg. 1990; 86:773-5. 